\title{
GLOBULAR CLUSTER SYSTEMS IN OTHER GALAXIES
}

\author{
WILLIAM E. HARRIS \\ Dept of Physics $\&$ Astronomy, McMaster University \\ Hamilton, ON L8S 4M1, Canada \\ (harris@physun.physics.mcmaster.ca)
}

A hypothesis or theory is clear, decisive, and positive, but it is believed by no one but the man who created it. Experimental findings, on the other hand, are messy, inexact things which are believed by everyone except the man who did that work.

$$
\text { Harlow Shapley }
$$

\section{Introduction}

Baade and Shapley would surely be surpised and delighted to see how far we have moved from the original view, half a century ago, of globular clusters as the paradigmatic Population II objects. Considerable evidence, accumulated especially over the last decade, now demonstrates that globular cluster systems (GCS's) make up a stellar population with distinctive characteristics of their own and are not just subsets of the old Pop II field stars that fill the halos of large galaxies. For example, the halo clusters have mean metallicities and metallicity distributions that are often unlike the field halo stars in the same region of their parent galaxy; the spatial structure of the GCS is often quite a bit shallower than the more centrallyconcentrated halo and spheroid population; and in some galaxies at least, the GCS is a dynamically and kinematically distinct entity within the halo.

For several years, the standard interpretation of all this has been that the globular clusters represented the oldest visible part of any large galaxy a sort of chronological link between the halo field stars that define the main spheroid of the galaxy, and the still older dark matter that first laid down the overall potential well of the protogalaxy. Now, however, new challenges to this undoubtedly simplistic picture are arising on several fronts, and it seems more likely than ever that properly designed observations of globular 
clusters are capable of telling us unique and startling things about the sequence of events during galaxy formation.

The systematic investigation of GCS's around galaxies has now grown prolifically a well defined observational subdiscipline of its own. As such, it has become far too large to encompass properly within a single short discussion, and here I will concentrate only on a few currently active issues relevant to stellar populations and galaxy formation. For broader treatments of this general subject, the reader is encouraged to see other recent reviews (Harris 1991, 1993).

\section{Metallicity Distributions: Where's the Ceiling?}

A particularly strong advantage of working with globular clusters in distant galaxies is that - unlike the field halo stars! - we can measure them one by one, and thus explicitly derive the distribution functions for such properties as metallicity, radial velocity, and mass (or luminosity). Several major studies of the metallicity distributions (MDFs) in large galaxies have recently appeared based on multicolor CCD photometry of large samples of clusters, and amount in total to nothing less than a revolution in this subject: M31 (Reed, Harris and Harris 1994), NGC 5128 (G. Harris et al. 1992), M87 (Couture, Harris and Allwright 1990; Lee and Geisler 1993; Harris, Harris and McLaughlin 1994), NGC 1399 (Bridges, Hanes and Harris 1991; Ostrov, Geisler and Forte 1993), NGC 3311 (Secker et al. 1994), and several Virgo ellipticals (Couture, Harris and Allwright 1991; Ajhar, Blakeslee and Tonry 1994). The work of Geisler and his colleagues with the sensitive broadband index $\left(C-T_{1}\right)_{0}$ has been especially effective here.

Some of these results are displayed in Fig. 1, for selected regions in three of the giant ellipticals: M87, NGC 1399, and NGC 3311. In all cases these MDFs are strikingly different from what we are used to seeing in the Milky Way or M31, where most clusters are quite metal-poor $(\langle[\mathrm{Fe} / \mathrm{H}]\rangle \simeq-1.6, \sigma[\mathrm{Fe} / \mathrm{H}]=0.3)$, with a less populous "disk cluster" component at $\langle[\mathrm{Fe} / \mathrm{H}]\rangle \simeq-0.5$ (e.g. Zinn 1985; Armandroff 1989; Reed et al. 1994). Single Gaussian-like functions are not good representations of any of these MDFs; in most cases two or three separate components are necessary (Ashman and Zepf 1992; Lee and Geisler 1993). In a generic way, these must represent fairly distinct epochs of cluster formation just as in the Milky Way, though these need not necessarily be due to the rather specific merger/shock mechanism promoted by Ashman and Zepf (1992) and Zepf and Ashman (1993).

Photometric metallicity indicators like $C-T_{1}$ have proven to be extremely efficient ways to derive MDFs, but a serious problem is that we urgently need a way to calibrate the upper end of the metallicity scale. The 


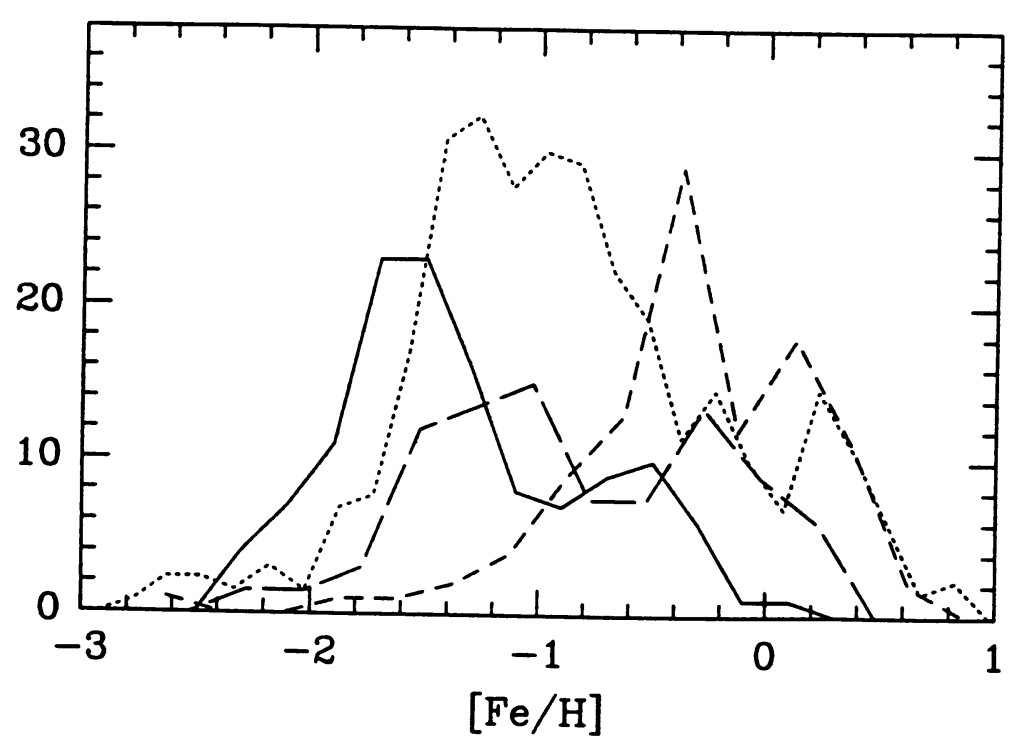

Figure 1. Metallicity distributions for the Milky Way and three giant E galaxies. Solid line: The Milky Way GCS. Dotted line: The mid-to-outer halo of M87 (Lee and Geisler, 1993). Dashed line: NGC 3311 (Secker et al., 1994). Long-dashed line: NGC 5128 (Harris et al., 1992).

reddest cluster colors cannot be translated accurately into $[\mathrm{Fe} / \mathrm{H}]$ values because they are well above solar metallicity, and our most metal-rich calibrators (the Milky Way bulge clusters) are at $[\mathrm{Fe} / \mathrm{H}] \lesssim-0.3$. The problem is discussed at length by G. Harris et al. (1992) and affects all the data mentioned above. The solution may well require combinations of spectroscopically based indices for the reddest objects, along with model cluster colors built from high-metallicity isochrones; but whatever the route, it needs attention if we are to understand the enrichment histories of these galaxies correctly.

All of us are used to thinking of globular clusters as metal-poor. But the "classic" metal-poor globulars in the halos of spiral galaxies and dwarfs are the exception rather than the rule; most of the globular clusters in the universe reside in giant $\mathrm{E}$ galaxies, and the MDFs in these galaxies are now telling us that most globular clusters have heavy-element abundances that are not terribly low - one-sixth solar or more. Furthermore, we do not really know where the top end of the MDF for globular clusters lies; it is certainly higher than $[\mathrm{Fe} / \mathrm{H}]=0$ and in many giant $\mathrm{E}$ galaxies it may extend up to three times solar metallicity or even more. Clearly these results pose a formidable challenge to any theoretical model of cluster formation which 
requires low-metallicity gas (cf. Fall and Rees 1985; Kang et al. 1990).

A topic which deserves mention here, although it is only indirectly connected to the MDF, is the issue of GCS dynamics. Pioneering attempts to measure radial velocities for some dozens of clusters in large $\mathrm{E}$ galaxies (e.g. H. Harris et al. 1988; Mould et al. 1990) first showed that the GCS's there had velocity dispersions generally larger than the underlying halo light. New data for the Fornax cD NGC 1399 (Grillmair et al. 1994; Arnaboldi et al. 1994) show, startingly, that the the GCS does not participate in the strong overall rotation characterizing the outer halo and thus that the globular clusters are a kinematically distinct (much older?) part of the halo. Tantalizing clues like this need to be pursued vigorously, now that we are in the era of sensitive multi-object spectrographs and the new 8- to 10-meter telescopes. If velocity samples of hundreds - even thousands - of globular clusters around these giant galaxies can be obtained, the GCS kinematics as a function of position and metallicity can be mapped out in great detail, and we can begin to study even the orbital distribution (complete with anisotropy), thus placing entirely new constraints on the formation history and dynamical evolution of these systems.

\section{Cooling Flows, Mergers, Specific Frequencies, and All That}

A large spiral galaxy like the Milky Way and M31 typically contains a couple of hundred globular clusters. A decent-sized normal elliptical might hold a couple of thousand. These totals pale in comparison to the truly enormous numbers of clusters - tens of thousands per galaxy - that populate some of the supergiant $\mathrm{cD}$ galaxies at the centers of rich Abell-type clusters (Virgo, Hydra I, Coma, and a few others; see Harris, Pritchet and McClure 1994 for a summary). The relative sizes of GCS populations are conventionally described by the specific frequency $S_{N}$ (Harris and van den Bergh 1981), which is the ratio of the total number of globular clusters to the galaxy luminosity. When it was discovered in the early 1980's that the giant $\mathrm{cD}$ galaxies were often surrounded by massive amounts of hightemperature gas and inward-moving "cooling flows", the idea was raised (Fabian, Nulsen and Canizares 1984) that perhaps the excess globular clusters present in such galaxies have been forming continuously out of the cooling-flow gas over the past Hubble time. This idea has been brought up again from time to time in the literature, but until recently there was not much specific data one could bring to bear either for or against it. However, a variety of new observations weigh strongly against this hypothesis:

- Harris et al. (1994) discuss the available GCS data for a dozen centralsupergiant $\mathrm{cD}$ galaxies in a wide variety of cluster environments. About half of them have the "high specific frequency" anomaly $\left(S_{N} \simeq 15\right.$; a 
typical Virgo elliptical has $S_{N} \sim 5$, and most $\mathrm{E}$ galaxies in small groups or the field have $S_{N} \sim 2$. See Harris 1991). But there is no correlation between GCS population size and any known feature of the intracluster gas (X-ray luminosity, gas temperature, total gas mass, or cooling flow rate). That is, some of these cD's (e.g. M87, NGC 3311) have huge cluster populations but relatively small X-ray halos, or vice versa (NGC 4874, UGC 9799); others (NGC 6166, and perhaps NGC 1275) have normal or subnormal GCS's but exceptionally massive amounts of intracluster gas. Since cooling flows are quite long-lived phenomena, taking many Gyr either to initiate or to die away, this suggests that cooling flows in these galaxies have not been relevant to globular cluster formation over the past several $10^{9} \mathrm{y}$. Interestingly, however, a rough correlation does exist between GCS size and the Bautz-Morgan class of the host Abell cluster: the cD's that are more centrally dominant tend to have less populous GCS's (McLaughlin, Harris and Hanes 1994). One interpretation of this correlation might be that the central galaxy started out with a relatively large GCS, but grew by accretion of many surrounding galaxies; since the neighboring galaxies would all have had much lower specific frequencies, the $S_{N}$ of the merged product would systematically decrease as more victims were accreted.

- Clusters that have condensed out of the quiet, inward-flowing gas, should all have plunging, radial orbits. Grillmair et al. (1994) have measured the velocity distribution of the halo clusters around the Fornax $\mathrm{cD}$ galaxy NGC 1399. They find that the velocity dispersion of the clusters near the characteristic radius of the cooling flow is far larger than would be expected under such a model; i.e. the cluster velocity distribution is more nearly isotropic.

- In most giant $\mathrm{E}$ galaxies the mean globular cluster metallicity is at the level $[\mathrm{Fe} / \mathrm{H}] \sim-0.8$ to -1.0 , except (see above) in the innermost few kpc. By contrast, the X-ray gas has a characteristic metaliicity 3 to 10 times higher (e.g. Rothenflug and Arnaud 1985; Forman et al. 1993).

All of this material suggests that the dilute, low-density, solar-metallicity gas within a cooling flow is simply the wrong place to look for globular cluster formation. The onset of a cooling flow is more likely to be a later event in the galaxy's history, generically unrelated to the presence of the older GCS (cf. the additional discussion of Harris et al. 1994).

The $\mathrm{cD}$ galaxies that have very high specific frequencies - M87 and the others like it - are often referred to as having "excess" populations of globular clusters, with the implied interpretation that they started with some 
normal-sized GCS but somehow acquired more globulars in a later, separate chain of events. But it is important to realize that there is nothing to distinguish the individual clusters in (say) M87 or NGC 1399 from those in other giant $\mathrm{E}$ galaxies, in terms of their luminosity distributions, metallicities, or the way in which they are distributed spatially around their parent galaxy (Harris 1991; Harris et al. 1991). They simply appear in larger numbers. It seems more nearly correct to assert that they were formed in the same original process that was at work in other protogalaxies, but operating at higher efficiency.

The formation of genuinely "young" stars and globular clusters may, however, be stimulated by mergers between gas-rich galaxies (Schweizer 1987). Star clusters with masses $\gtrsim 10^{5} M_{\odot}$ and ages much less than $10^{9} \mathrm{yr}$ have been found in the central regions of active galaxies as diverse as NGC 3597 (Lutz 1991), NGC 1275 (Holtzman et al. 1992), NGC 7252 (Whitmore et al. 1993), and He 2-10 (Conti and Vacca 1994). The accumulated evidence makes a highly convincing case that the young globular clusters in these locations formed as a direct result of the merger or starburst events that we see happening in front of us.

Ashman and Zepf (1992) and Zepf and Ashman (1993) have taken this scenario further to suggest that the rich cluster populations in $\mathrm{E}$ galaxies formed as a result of mergers. Their model directly attacks the classic "specific frequency problem" that has been a long-standing stumbling block to the idea that elliptical galaxies in general formed by merging of pre-existing disk galaxies (Toomre 1977). The nature of the problem is simple: large $\mathrm{E}$ galaxies have specific frequencies $S_{N} \sim 5$ (or higher), substantially larger than the $S_{N} \sim 1$ level that characterizes spirals like M31 and the Milky Way (Harris 1991). How can disk galaxies that have dozens or hundreds of old-halo clusters then combine to make ellipticals that have thousands? Several authors have recently claimed that active cluster formation during mergers will increase $S_{N}$ in the merger product and thus permit this approach to work after all (e.g. Schweizer 1987; Lutz 1991; Holtzman et al. 1992; Whitmore et al. 1993).

Unfortunately these claims are, in general, wrong. The reason is that both star clusters and new field stars form in the gas clouds, and the quantity that determines the specific frequency is the ratio of cluster mass to fieldstar mass - in other words, the efficiency of cluster formation. $S_{N}$ could increase, stay the same, or even decrease depending on what fraction of the gaseous material gets compressed into the high-density clumps that eventually emerge as bound star clusters. There is no guarantee that the merger processes we see now are any more efficient at cluster formation than what went on in the early protogalactic epoch, when there was much more gas. 
The nature of this problem is correctly stated by Ashman and Zepf (1992) and algebraically formulated. However, I am not aware of any discussion of the actual numerical implications, which turn out to be quite severe if we want to build large $\mathrm{E}$ galaxies this way. A simple representative example will demonstrate the point. Denote the formation efficiency as $E=M_{c l} / M_{g}$, where $M_{g}$ is the mass of gas available for star formation and $M_{c l}$ is the stellar mass that ends up in bound star clusters. Zepf and Ashman (1993) present a thorough summary of the data for several "merger" galaxies which show repeatedly that $E$ is near $1 \%$ in such systems. This is encouragingly close to the estimate of a few times $10^{-3}$ for star clusters forming in giant molecular clouds in the disk of the Milky Way (Larson 1990b; Harris and Pudritz 1994. In the notation of Harris and Pudritz, $E=\eta \cdot c$, where $\eta$ is the average ratio of protocluster core mass to GMC mass, and $c$ is the typical number of protocluster cores embedded in a GMC). In other words, it is extremely difficult to build a bound star cluster, and most stars form within smaller clumps and associations that quickly become unbound. In a protocluster, gas must be converted to stars at $\gtrsim$ $50 \%$ efficiency, and the observations suggest that such levels are achieved only within the densest cores of GMCs (Larson 1988, 1990b).

The "average" mass of a globular cluster is $M_{\oplus} \equiv M_{T} / N_{T}$, where $M_{T}$ is the total mass in the GCS and $N_{T}$ the total number of clusters; for a standard mass-to-light ratio $M / L_{V}=2$ and the cluster mass distribution function as given by Harris and Pudritz (1994; see their eqs. 4.4 and 4.5), we obtain $M_{\oplus} \sim 3 \times 10^{5} M_{\odot}$. In contemporary disk galaxies, the amount of molecular gas present differs widely, but representative amounts observed in interacting systems (IRAS galaxies, systems like NGC 1275, etc.) are $M_{g} \sim 10^{10} M_{\odot}$ (e.g. Sanders et al. 1985, 1986; Lazareff et al. 1989). Thus, for $E=0.01$, we could expect to find $\gtrsim 300$ clusters forming from a typical merger. In fact, because most clusters are at the low-mass end $(d N / d M \sim$ $M^{-1.7}$; cf. Harris and Pudritz), only the top few dozen of these would be more luminous than the average and thus visible in galaxies at large distances. These totals are encouragingly close to the numbers of young clusters we see in NGC 1275,3597 , and 7252 .

For comparison, let us take a normal giant elliptical like NGC 4472, which has $S_{N}=5$ and a GCS population of $\sim 7000$ clusters (Harris 1991, Table 1). To build such a system at the same $\sim 1 \%$ efficiency would require an initial gas reservoir $M_{g} \gtrsim 2 \times 10^{11} M_{\odot}$. More realistically, we should probably require $M_{g} \sim 10^{12} M_{\odot}$, since (a) much of the gas - perhaps half - does not collect into the large giant molecular clouds within which dense protoclusters can form; and (b) the amount of mass going into in the GCS was probably at least twice as high as we see now, once we account for the long-term effects of cluster erosion by dynamical friction, tidal shocking, 
and tidal evaporation. The only epoch during which such huge amounts of gas were routinely available within galaxies was the protogalactic era itself. Indeed, Ashman and Zepf's (1992) discussion implicitly suggests this conclusion, i.e. that their disks which merge to form ellipticals were mostly, if not all, gaseous. However, it is not clear to me how this scenario then differs from the classic picture of galaxy formation out of gaseous "fragments" (Searle 1977; Searle and Zinn 1978).

In short, I suggest that the merger events we see going on in the presentday universe are capable of adding to GCS's in a minor way, but the vast numbers of globular clusters residing in giant ellipticals must have been built in during the primary (and early!) epoch of galaxy formation. The true importance of the events going on in sites like NGC 1275 or NGC 7252 is that they give us a direct glimpse of the process of massive cluster formation which went on wholesale $15 \mathrm{Gyr}$ ago. On the other hand, later mergers between disk galaxies remain a plausible source of the large $\mathrm{E}$ galaxies in small groups which have rather low specific frequencies, $S_{N} \lesssim 2$ (Harris 1981). It is likely that this is exactly what an object like NGC 7252 will turn into, once the tidal debris and merger-induced star formation have settled down.

\section{Cluster Formation: When and How?}

Most of the globular clusters in the universe reside in giant $\mathrm{E}$ galaxies. However, similarly massive, old clusters can be found (albeit in far smaller numbers) in the halos of spiral galaxies, dwarf ellipticals, and even some irregulars. Because the globular clusters in all these environments are far more similar to one another than their parent galaxies are, it is highly likely that they represent a common thread in the early history of galaxies. What do the data tell us about pinning down their main epoch of formation, and are we now in a position to construct an accurate formation model?

It is scarcely possible to improve on the concise statement by Larson (1990a): "None of [the] evidence excludes the more conventional view that most elliptical galaxies ... were formed at early times by the dissipative collapse of clumpy protogalaxies." As the data discussed in the preceding sections indicate, the globular cluster populations and their metallicity distributions now provide particularly strong evidence that most $\mathrm{E}$ galaxies did not form from the mergers of things like present-day disk galaxies, even though some merging of fully-formed galaxies is obviously going on today.

\section{References}

Ajhar, E.A., Blakeslee, J.P. and Tonry, J.L., 1994, preprint

Armandroff, T.E., 1989, A. J. 97, 375 
Arnaboldi, M., Freeman, K.C., Saha, P., Capaccioli, M., Ford, H., Frillmiar, C. and Hui, X., 1994, B. A. A. S. 26, 941

Ashman, K.M. and Zepf, S.E,. 1992, Ap. J. 384, 50

Bridges, T.J., Hanes, D.A. and Harris, W.E., 1991, A. J. 101, 469

Couture, J., Harris, W.E. and Allwright, J.W.B., 1990, Ap. J. Suppl. 73, 671

Couture, J., Harris, W.E. and Allwright, J.W.B., 1991, Ap. J. 372, 97

Conti, P.S. and Vacca, W.D., 1994, Ap. J. 423, L97

Fall, S.M. and Rees, M.J., 1985, Ap. J. 298, 18

Grillmair, C.J., Freeman, K.C., Bicknell, G.V., Carter, D., Couch, W.J., Sommer-Larsen, J. and Taylor, K., 1994, Ap. J. 422, L9

Harris, G.L.H., Geisler, D., Harris, H.C. and Hesser, J.E., 1992, A. J. 104, 613

Harris, W.E., 1991, Ann. Rev. Astron. Astroph. 29, 543

Harris, W.E., 1993, The Globular Cluster - Galaxy Connection, ASP Conf. Series, 48, ed. G.H.Smith and J.P.Brodie, San Francisco: ASP, 48, 472

Hesser, J.E., 1993, Structure and Dynamics of Globular Clusters, ASP Conf. Series 50, ed. S.G.Djorgovski and G. Meylan, San Francisco: ASP, p.15

Holtzman, J. et al., 1992, A. J. 103, 691

Kang, H., Shapiro, P., Fall, S.M. and Rees, M.J., 1990, Ap. J. 363, 488

Laird, J.B., Rupen, MP., Carney, B.C. and Latham, D.W., 1988, A. J. 96, 1908

Larson, R.B., 1988, Globular Cluster Systems in Galaxies, IAU Symposium 126, ed J.E.Grindlay and A.G.D.Philip, Dordrecht: Reidel, p.311

Larson, R.B., 1990a, P. A. S. P. 102, 709

Larson, R.B., 1990b, Physical Processes in Fragmentation and Star Formation, ed. R.Capuzzo-Dolcetta, C.Chiosi and A.DiFazio, Dordrecht: Kluwer, p.389

Lazareff, B., Castets, A., Kim, D.-W. and Jura, M., 1989, Ap. J. 336, L13

Lee, M.G. and Geisler, D., 1993, A. J. 106, 493

Lutz, D., 1991, A. \& A. 245, 31

Ostrov, P., Geisler, D. and Forte, J.C., 1993, A. J. 105, 1762

Sanders, D.B., Scoville, N.Z. and Solomon, P.M., 1985, Ap. J. 289, 373

Sanders, D.B., Scoville, N.Z., Young, J.S., Soifer, B.T., Schloerb, F.P., Rice, W.L. and Danielson, G.E., 1986, Ap. J. 305, L45

Whitmore, B., Schweizer, F., Leitherer, C., Borne, K. and Robert, C., 1993, A. J. 106, 1354

Zepf, S.E. and Ashman, K.M., 1993, M. N. R. A. S. 264, 611

Zinn, R., 1985, Ap. J. 293, 424

RICH: How sure are you that you have identified proto-globular clusters, taking into account (1) mass-loss by the massive stars, and (2) the probable absence of low-mass stars $(M$ below $\approx 2)$ ? By your criterion, a globular cluster must retain at least $10^{5} M_{\odot}$ over the course of cosmic time. Can you demonstrate that your proto-globulars meet your criterion?

HARRIS: The bright, blue objects discovered in NGC 1275,7252 , etc. certainly seem massive enough to be more than $10^{5} M_{\odot}$ and thus are good candidates for young globular clusters (by the way, I'd prefer to use the term "proto-globular" for the progenitor gas cloud that gives rise to the cluster; but once the gas has formed into stars and it hasn't dissolved into the field, then it's entitled to be called a star cluster!). To see the evidence for the masses of these young objects, one should see the papers by Holtzman et al. (1992) and Whitmore et al. (1993). However, I think it's quite likely that not all of these young objects (particularly the lowest-mass ones) will be destroyed as time goes on, by a wide variety of dynamical destructive processes. So it's only after a longer period of time that we will know who the survivors will be. 
RENZINI: I have two questions: (1) What would you infer from your wide metallicity distribution of globulars in E-galaxies about the metallicity distribution of the unclustered stars in these galaxies? You know, it has been claimed from population synthesis methods that E-galaxies would be single metallicity objects. (2) Do Magellanic Cloud globulars fit the $M^{-1.7}$ distribution?

HARRIS: (1) For the field-star population, in analogy with M31, I would not be surprised if the MDF in large E-galaxies were more metal-rich than the clusters on average, but somewhat narrower in dispersion. However, I also think the best way to answer this is to use the imaging capabilities of HST to observe the MDF directly for the brightest red-giant stars in the nearest giant ellipticals. It's within reach of HST and should be done!

(2) Yes, it's quite consistent with that distribution, although the statistical confidence is of course much lower (since there are only a dozen or so LMC globulars). ALCAINO: The luminosity function of the Galactic globular clusters shows a Gaussian distribution with a peak at $\mathrm{M}_{V}=-7.5$. Would you comment if it is correct to state that our current knowledge shows for all the globular cluster systems, independent of the parent galaxy type and total luminosity, as well the peak of the Gaussian at $M_{V}=-7.5 \pm 0.5$, and if so, if this is now widely used as standard candle.

HARRIS: All the new data reinforce the similarity of the turnover luminosity in large galaxies of all types. The galaxy-to-galaxy scatter in this quantity is $0.2-0.3$ magnitude -not small enough to make it a first-rate standard candle at the level of Cepheids or planetary nebulae, but useful. However, the real importance of the uniformity of this mass distribution is, I think, the strong constraint it is going to exert on cluster formation modelling in different types of galaxies.

DE BOER: I have two comments on metallicity. You (and others) show diagrams giving $[\mathrm{Fe} / \mathrm{H}]$. This is allowed only if you have seen iron lines; otherwise one should use "metallicity index" or so, which may be on (based on or identical to) an $[\mathrm{Fe} / \mathrm{H}]$ scale. The improper use of $[\mathrm{Fe} / \mathrm{H}]$ causes a lot of confusion!

HARRIS: I agree, although many others in this room will have to plead equally guilty!

DE BOER: My second comment deals with the calibration as you also addressed. The calibration of colour index and metallicity is very important indeed as well as very difficult. During a JD at the Baltimore General Assembly the (B-V) versus metallicity calibration of the globular clusters by Zinn and West (1984, Ap.J. 55, 45) was mentioned and it was stressed that (B-V) does not translate one-to-one into metallicity. That discussion has been published (de Boer, 1988, in Proc. JCM5 and Comm 37/3 at XX-th General Assembly, Ed. G. Cayrel, Obs. de Paris, p.35). The problem lies in how the HB is populated with stars. Of course, $(C-T)$ is a better metallicity index than (B-V), but it has to be verified if it works also for star clusters (which I doubt). Such a calibration must be done carefully and it will take a lot of effort. Until that time I see no reason to believe any metallicity of globular clusters in/near giant ellipticals.

HARRIS: You're quite right that any color index for the integrated light of a stellar system will have some genuine scatter built into it because of the way the HR diagram is filled up in detail (i.e. the second parameter problem). Also, (B-V) is not the most effective choice because it has a rather low systematic change with metallicity, not because of any particular feature of the intrinsic scatter. However, I also think you are being entirely too skeptical if you want to throw out the material for the clusters in the giant ellipticals entirely. The advantage of an index like 
$\left(\mathrm{C}-\mathrm{T}_{1}\right)$ or (B-I) is that they have an enormous range in color (i.e. sensitivity to metallicity) and thus minimize the effect of the intrinsic scatter and observational error. There seems little question that what such an index measures for any old cluster is, primarily, metallicity (see the paper by Geisler and Forte, Ap.J. Letters 1991 for the (C- $\left.\mathrm{T}_{1}\right)$ calibration). The main problem area, for me, is what to do at the high-metallicity end of the scale to translate a color index into a correct abundance index.

MINITTI: If in general field stars are more metal rich than globulars, this galaxy NGC 3311 must have a remarkable field star population! Can you comment on this?

HARRIS: The halo light in NGC 3311 is slightly redder even than the clusters, so at face value it should be still more metal-rich. Perhaps it means that such galaxies may have nothing much in it with less than solar metallicity, even far out in the halo. This goes against traditional impressions that every galaxy ought to have some amount of old, metal-poor stars in them, but that may not be true at all for giant ellipticals. 